\title{
Genes involved in oxidative phosphorylation are coordinately upregulated with fasting hyperglycaemia in livers of patients with type 2 diabetes
}

\author{
H. Misu • T. Takamura $\cdot$ N. Matsuzawa $\cdot$ A. Shimizu \\ T. Ota • M. Sakurai $\cdot$ H. Ando $\cdot$ K. Arai - T. Yamashita • \\ M. Honda $\cdot$ T. Yamashita $\cdot$ S. Kaneko
}

Received: 30 May 2006 / Accepted: 19 September 2006/ Published online: 23 December 2006

(C) Springer-Verlag 2006

\begin{abstract}
Aims/hypothesis Mitochondrial oxidative phosphorylation (OXPHOS) plays an important role in the pathophysiology of type 2 diabetes. Genes involved in OXPHOS have been reported to be down-regulated in skeletal muscle from patients with type 2 diabetes; however, hepatic regulation is unknown. Materials and methods We analysed expression of genes involved in OXPHOS from the livers of 14 patients with type 2 diabetes and 14 subjects with NGT using serial analysis of gene expression (SAGE) and DNA chip analysis. We evaluated the correlation between expression levels of genes involved in OXPHOS and the clinical parameters of individuals with type 2 diabetes and NGT.

Results Both gene analyses showed that genes involved in OXPHOS were significantly upregulated in the type 2 diabetic liver. In the SAGE analysis, tag count comparisons of mitochondrial transcripts showed that ribosomal RNAs (rRNA) were 3.5-fold over-expressed, and mRNAs were 1.2-fold over-expressed in the type 2 diabetes library. DNA chip analysis revealed that expression of genes involved in OXPHOS, which correlated with several nuclear factors, including estrogen-related receptor- $\alpha$ or peroxisome proliferator-activated receptor- $\gamma$, was a predictor of fasting plasma glucose levels, independently of age, BMI, insulin
\end{abstract}

Electronic supplementary material Supplementary material is available in the online version of this article at http://dx.doi.org/ $10.1007 / \mathrm{s} 00125-006-0489-8$ and is accessible to authorised users.

H. Misu $\cdot$ T. Takamura $(\bowtie) \cdot$ N. Matsuzawa $\cdot$ A. Shimizu

T. Ota $\cdot$ M. Sakurai $\cdot$ H. Ando $\cdot$ K. Arai $\cdot$ T. Yamashita $\cdot$

M. Honda $\cdot$ T. Yamashita $\cdot$ S. Kaneko

Deparment of Disease Control and Homeostasis,

Kanazawa University Graduate School of Medical Science,

13-1 Takara-machi, Kanazawa,

Ishikawa 920-8641, Japan

e-mail: ttakamura@m-kanazawa.jp resistance and fasting insulin levels $(p=0.04)$. Surprisingly, genes involved in OXPHOS did not correlate with peroxisome proliferator-activated receptor- $\gamma$ coactivator- $1 \alpha$ or nuclear respiratory factor 1 .

Conclusions/interpretation Our results indicate that upregulation of genes involved in OXPHOS in the liver, which are regulated by different mechanisms from genes in the skeletal muscle, is associated with fasting hyperglycaemia in patients with type 2 diabetes.

Keywords DNA chip - Liver - Oxidative phosphorylation . Serial analysis of gene expression · Type 2 diabetes

\begin{tabular}{|c|c|}
\hline \multicolumn{2}{|l|}{ Abbreviations } \\
\hline ESRRA & oestrogen-related receptor- $\alpha$ \\
\hline NRF1 & nuclear respiratory factor 1 \\
\hline OXPHOS & oxidative phosphorylation \\
\hline OXPHOS-CR & oxidative phosphorylation coregulated \\
\hline PCK & phosphoenol-pyruvate carboxykinase \\
\hline PPARGC1A & $\begin{array}{l}\text { peroxisome proliferator-activated } \\
\text { receptor- } \gamma \text { coactivator- } 1 \alpha\end{array}$ \\
\hline PPARG & $\begin{array}{l}\text { peroxisome proliferator-activated } \\
\text { receptor- } \gamma\end{array}$ \\
\hline rRNA & ribosomal RNA \\
\hline SAGE & serial analysis of gene expression \\
\hline THRA & thyroid hormone receptor $\alpha$ \\
\hline
\end{tabular}

\section{Introduction}

Type 2 diabetes is caused by defects in insulin responsiveness of the skeletal muscle and liver, as well as glucosestimulated insulin secretion by pancreatic beta cells [1]. It is hypothesised that these features of type 2 diabetes are 
caused by mitochondrial dysfunction [2]. Mitochondria in the skeletal muscle of patients with type 2 diabetes have ultrastructural changes and an impaired bioenergetic capacity [3]. Recently, several reports [4-7] have suggested that impairment of mitochondrial oxidative phosphorylation (OXPHOS) in the skeletal muscle contributes to type 2 diabetes. Petersen et al. reported that insulin resistance in the skeletal muscle of insulin-resistant offspring of patients with type 2 diabetes is associated with dysregulation of intramyocellular fatty acid metabolism, possibly because of an inherited defect in mitochondrial OXPHOS [4, 5]. Moreover, genes involved in OXPHOS are downregulated in the skeletal muscle of patients with type 2 diabetes, and peroxisome proliferator-activated receptor- $\gamma(P P A R G)$ coactivator-1 $\alpha$ (PPARGC1A, also known as $P G C-1 \alpha$ ) has been identified as a candidate master gene for controlling expression of genes involved in OXPHOS in skeletal muscle $[6,7]$.

The liver is a key organ in glucose homeostasis. In the fasting state, normoglycaemia is maintained by hepatic gluconeogenesis. Type 2 diabetes is characterised by the impairment of insulin's ability to inhibit gluconeogenesis in the liver. The rate of gluconeogenesis is controlled by key enzymes, including phosphoenolpyruvate carboxykinase (PCK) and glucose-6-phosphatase (G6PC), in response to environmental and hormonal stimuli. Transcriptional activation of the PEPCK promoter requires coactivation of glucocorticoid receptors and the liver-enriched transcription factor hepatic nuclear factor- $4 \alpha$ (HNF4A) by PGC- $1 \alpha$, indicating that PGC- $1 \alpha$ is a key regulator of gluconeogenesis as well as of OXPHOS [8-10].

Contrary to the decrease of PPARGC1A expression in the skeletal muscle of patients with type 2 diabetes, PPARGC1A expression in the liver has been shown to be elevated in animal models of diabetes [8-11]. Tissue-specific regulation of metabolic pathways through PPARGC1A may take place in the skeletal muscle and liver. Thus, this differential expression might induce coordinated upregulation of genes involved in OXPHOS in the liver of patients with type 2 diabetes. To examine this hypothesis, we investigated comprehensive gene expression profiles in the liver of patients with type 2 diabetes and control subjects with NGT using the serial analysis of gene expression (SAGE) technique [12] and DNA chip analysis.

\section{Subjects and methods}

Samples Samples for SAGE and real-time quantitative polymerase chain reaction analyses were obtained from five patients with type 2 diabetes and five subjects with NGT, all of whom had undergone surgical treatments for malignant tumours, such as gastric cancer, gall bladder cancer and colon cancer. Hepatic tissues were surgically removed from the non-cancerous part of the liver and frozen in liquid nitrogen. We confirmed the absence of microscopic metastasis in liver samples histologically. There were no significant differences in age, BMI and liver function tests between the two patient groups (Table 1). Of the five patients with diabetes, three were treated with diet therapy alone and two were treated with insulin therapy.

Samples for DNA chip analysis were obtained from nine patients with type 2 diabetes and nine subjects with NGT, who were admitted to Kanazawa University Hospital from April 2000 to March 2003. Hepatic tissues were obtained with percutaneous needle liver biopsy, and immediately frozen in liquid nitrogen and stored at $-80^{\circ} \mathrm{C}$ until use. There were no significant differences in age, BMI, liver function tests and homeostasis model assessment of insulin resistance between the two patient groups (Table 2). Patients with type 2 diabetes were treated with diet therapy alone or insulin; no patients were receiving any oral hypoglycaemic agent. Participants receiving statins, ACE inhibitors or angiotensin II receptor blockers were excluded.

All subjects tested negative for hepatitis $\mathrm{B}$ and $\mathrm{C}$ viruses. No subjects experienced body weight loss or malnutrition before liver biopsies. All liver biopsies were performed in the fasting state. Patients with type 2 diabetes were diagnosed on the basis of criteria established by an expert committee on the diagnosis and classification of diabetes mellitus [13]. Subjects with NGT were diagnosed on the basis of fasting plasma glucose values $<6.1 \mathrm{mmol} / 1$ and glycosylated haemoglobin $<6.5 \%$. All patients provided written informed consent for this study. The experimental protocol was approved by the relevant ethics committee in our institution, and was carried out in accordance with the Declaration of Helsinki.

Table 1 Clinical and biochemical characteristics of patients with NGT and type 2 diabetes for SAGE analysis

\begin{tabular}{lll}
\hline & NGT & Type 2 diabetes \\
\hline$n$ & 5 & 5 \\
Age (years) & $62.6 \pm 11.8$ & $60.2 \pm 11.0$ \\
Sex (men/women) & $3 / 2$ & $3 / 2$ \\
BMI (kg/m $\left.{ }^{2}\right)$ & $21.7 \pm 4.4$ & $25.2 \pm 5.2$ \\
Random plasma glucose (mmol/l) & $6.3 \pm 0.3$ & $9.5 \pm 3.0^{*}$ \\
$\mathrm{HbA}_{1 \mathrm{c}}(\%)$ & $5.2 \pm 1.1$ & $8.1 \pm 2.0^{*}$ \\
$\mathrm{AST}(\mathrm{IU} / \mathrm{l})$ & $34 \pm 18$ & $28 \pm 24$ \\
$\mathrm{ALT}(\mathrm{IU} / \mathrm{l})$ & $28 \pm 21$ & $29 \pm 18$ \\
$\gamma \mathrm{GTP}(\mathrm{IU} / \mathrm{l})$ & $19 \pm 7$ & $44 \pm 24$ \\
Total cholesterol (mmol/l) & $4.1 \pm 0.6$ & $4.8 \pm 1.0$ \\
Triacylglycerol $(\mathrm{mmol} / \mathrm{l})$ & $1.2 \pm 0.6$ & $1.5 \pm 0.8$ \\
\hline
\end{tabular}

Data are mean $\pm \mathrm{SD}$

AST Aspartate transaminase, ALT alanine aminotransferase, $\gamma G T P$ gamma-glutamyltransferase

$* p<0.05$ vs NGT 
Table 2 Clinical and biochemical characteristics of patients with NGT and type 2 diabetes in DNA chip analysis group

\begin{tabular}{|c|c|c|}
\hline & NGT & Type 2 diabetes \\
\hline$n$ & 9 & 9 \\
\hline Age (years) & $42.6 \pm 4.6$ & $52.3 \pm 10.6$ \\
\hline Sex (men/women) & $6 / 3$ & $6 / 3$ \\
\hline BMI $\left(\mathrm{kg} / \mathrm{m}^{2}\right)$ & $26.7 \pm 4.9$ & $24.7 \pm 4.5$ \\
\hline Duration of diabetes (years) & 0 & $12.6 \pm 7.6^{*}$ \\
\hline Fasting plasma glucose $(\mathrm{mmol} / \mathrm{l})$ & $4.9 \pm 0.6$ & $7.7 \pm 1.7 *$ \\
\hline $\mathrm{HbA}_{1 \mathrm{c}}(\%)$ & $5.1 \pm 0.5$ & $7.3 \pm 0.5^{*}$ \\
\hline Fasting IRI & $8.4 \pm 4.0$ & $11.0 \pm 9.9$ \\
\hline HOMA-IR & $2.2 \pm 1.0$ & $3.9 \pm 3.6$ \\
\hline QUICKI & $0.36 \pm 0.024$ & $0.33 \pm 0.045$ \\
\hline $\operatorname{MCR}\left(\mathrm{mg} \mathrm{kg}^{-1} \min ^{-1}\right)$ & $7.7 \pm 2.2$ & $5.9 \pm 4.9$ \\
\hline AST (IU/1) & $39 \pm 20$ & $28 \pm 15$ \\
\hline ALT (IU/l) & $88 \pm 81$ & $43 \pm 37$ \\
\hline Total cholesterol $(\mathrm{mmol} / \mathrm{l})$ & $5.5 \pm 1.5$ & $5.3 \pm 0.6$ \\
\hline Triacylglycerol (mmol/l) & $1.7 \pm 1.2$ & $1.0 \pm 0.5$ \\
\hline HDL-cholesterol (mmol/1) & $1.1 \pm 0.2$ & $1.4 \pm 0.3$ \\
\hline
\end{tabular}

Data are mean $\pm \mathrm{SD}$

IRI Immunoreactive insulin, HOMA-IR homeostasis model assessment, QUICKI quantitative insulin sensitivity check index, $M C R$ glucose metabolic clearance rate, $A S T$ aspartate transaminase; $A L T$ alanine aminotransferas

$* p<0.05$ vs NGT

Serial analysis of gene expression ( $S A G E$ ) Total RNA was extracted from the homogenised samples using an isolation kit (ToTTALY RNA, Ambion, Austin, TX, USA). Polyadenylated RNA was extracted using a kit (Micropoly [A] Pure; Ambion). A total of $2.5 \mu \mathrm{g}$ of RNA in each sample was used for SAGE. The SAGE protocol has been previously described $[14,15]$. SAGE libraries were sequenced at random using a DNA sequencer (ABI PRISM 377; PE Biosystems, Foster City, CA, USA) and sequence kit (BigDye Terminator Cycle Sequence Kit; PE Biosystems). An NGT library was constructed with the original SAGE protocol (using ten base pair sequence tags), whereas the library for patients with type 2 diabetes was constructed with the long SAGE method (using 17 base pair sequence tags) [16]. Sequenced files were analysed with the SAGE 2000 software (http://www.sagenet.org). To minimise the effects of possible sequencing errors, only tags represented at least twice in each library were considered for analysis. The transcript identity of each SAGE tag in the type 2 diabetes library was obtained using the nucleotide-nucleotide basic local alignment search tool (BLAST) (http:// www.ncbi.nlm.nih.gov/BLAST/). The transcript identity of each SAGE tag in the NGT library was obtained by matching the unitag list against the human tag-to-gene 'reliable map' in SAGEmap (ftp://ftp.ncbi.nlm.nih.gov/pub/ sage/map/). The gene expression profiles of the type 2 diabetes and NGT libraries were compared through normalisation to $1,000,000$ tags, and statistical significance between libraries was calculated with SAGE 2000 software. All gene ontology annotations were gathered using Unigene cluster IDs collected from the SOURSE database (http://www. genome-www5.stanford.edu/cgi-bin/source/sourceSearch). For the analysis of the cellular component, the different gene ontology categories were further regrouped into nine classes (extracellular, mitochondrion, nucleus, cytoplasm, plasma membrane, ribosome, endoplasmic reticulum, Golgi apparatus, and others), using the definitions described by the Gene Ontology Consortium (http://www.geneontology.org/). The transcripts corresponding to the class 'mitochondria' included mitochondrial transcripts and nuclear transcripts coding proteins transferred to mitochondria. The class 'others' included transcripts in which the cellular component was unknown.

Additional details on methods For details on laboratory studies, DNA chip analyses, real-time PCR and statistical analyses, see Electronic supplementary material (ESM).

\section{Results}

SAGE expression profiles We obtained a total of 144,901 tags from the two libraries (NGT, 100,621 tags; type 2 diabetes, 44,280 tags). Many tags were repeatedly presented, and a total of 37,054 genes were unique in the two libraries (NGT, 27,622 genes; type 2 diabetes, 15,337 genes). To avoid counting tags caused by sequence error, tags counted only once in the library were eliminated. Tags found more than twice in the NGT and type 2 diabetes libraries represented 9,948 and 7,126 transcripts, respectively.

The 20 most abundant genes in each library are shown in Tables 3 and 4. Mitochondrial transcripts such as $16 \mathrm{~S}$ ribosomal RNA (rRNA) and cytochrome c oxidase subunit III occupied a high rank in the type 2 diabetes library, whereas transcripts encoding secretory proteins such as apolipoprotein C-I and albumin occupied a high rank in the NGT library.

Gene ontology cellular components of identified transcripts The gene ontology cellular components of all identified transcripts were gathered and regrouped into the nine representative classes designated by the Gene Ontology consortium (http://www.geneontology.org/) as shown in Fig. 1. The top class for the type 2 diabetes library was 'mitochondria'. The transcripts corresponding to the class included mitochondrial and nuclear transcripts coding proteins transferred to mitochondria. For instance, the mitochondrial transcripts included those coding 12S rRNA, 16S rRNA, cytochrome c oxidase subunit III, and ATP synthase F0 subunit 6 . The nuclear transcripts coding 
Table 3 The 20 most abundant genes and tags in the library of patients with NGT

\begin{tabular}{|c|c|c|c|c|}
\hline Tag sequence & UnigeneID & Description & Absolute tags & Tags per million \\
\hline TGGCCCCAGG & Hs. 268571 & Apolipoprotein C-I & 3,286 & 32,657 \\
\hline CTGGCCTCCC & Hs.73849 & Apolipoprotein C-III & 1,832 & 18,207 \\
\hline AGAATAAGAG & Hs.418167 & Albumin & 1,214 & 12,065 \\
\hline TGGACGCGCT & Hs.93194 & Apolipoprotein A-I & 1,144 & 11,369 \\
\hline GGAAAAGTGG & & Multiple match & 1,074 & 10,674 \\
\hline CCCATCGTCC & & Cytochrome c oxidase subunit II, mitochondrial & 989 & 9,829 \\
\hline AGCCCTACAA & & NADH dehydrogenase subunit 3 , mitochondrial & 936 & 9,302 \\
\hline CACCTAATTG & & ATP synthase F0 subunit 6 , mitochondrial & 894 & 8,885 \\
\hline GTGAAACCCC & & Multiple match & 872 & 8,666 \\
\hline CAAGCATCCC & & $12 \mathrm{~S}$ ribosomal RNA, mitochondrial & 813 & 8,080 \\
\hline CCTGTAATCC & & Multiple match & 760 & 7,553 \\
\hline TGTGGAGAGC & Hs. 237658 & Apolipoprotein A-II & 707 & 7,026 \\
\hline GTGACCACGG & & ESTs & 668 & 6,639 \\
\hline АСТTTТTCAА & & Multiple match & 661 & 6,569 \\
\hline GCCGGGCCCT & Hs. 2257 & Vitronectin & 598 & 5,943 \\
\hline TTCATACACC & & NADH dehydrogenase subunit 4 , mitochondrial & 534 & 5,307 \\
\hline CCACTGCACT & Hs. 193044 & Potassium inwardly-rectifying channel, subfamily $\mathrm{J}$, member 5 & 513 & 5,098 \\
\hline ACCCTTGGCC & & NADH dehydrogenase subunit 1 , mitochondrial & 483 & 4,800 \\
\hline GTGAAACCCT & & Multiple match & 467 & 4,641 \\
\hline TGTACCTCAG & & Multiple match & 455 & 4,522 \\
\hline
\end{tabular}

Genes were ranked by their absolute tag counts. We included the transcripts encoded by mitochondrial DNA in the library, such as cytochrome c oxidase subunit II and NADH dehydrogenase subunit III. The corresponding tags per million are shown to allow comparisons

products transferred to mitochondria included those coding cytochrome c oxidase subunit VIc, butyryl coenzyme A synthetase 1 and the aldehyde dehydrogenase 2 family. On the other hand, the top class for the NGT library was 'extracellular,' including genes encoding secretory proteins such as apolipoprotein C-I, apolipoprotein C-II, and albumin.
Expression of transcripts involved in OXPHOS in the SAGE library Of the transcripts associated with mitochondria, we focused on those involved in OXPHOS. By reference to a pathway gene list reported by Netaffx Analysis Center (http://www.affymetrix.com/index.affx), we found $53 \mathrm{nu}-$ clear transcripts involved in OXPHOS in our two libraries

Table 4 The 20 most abundant genes and tags in the library of patients with type 2 diabetes mellitus

\begin{tabular}{|c|c|c|c|c|}
\hline Tag sequence & UnigeneID & Description & Absolute tags & Tags per million \\
\hline CTAAGACTTCACCAGTC & & 16S ribosomal RNA, mitochondrial & 1,220 & 27,552 \\
\hline TGATTTCACTTCCACTC & & Cytochrome c oxidase subunit III, mitochondrial & 1,039 & 23,464 \\
\hline AGAATAAGAGAAAGAAA & Hs.418167 & Albumin & 774 & 17,480 \\
\hline CAAGCATCCCCGTTCCA & & $12 \mathrm{~S}$ ribosomal RNA, mitochondrial & 733 & 16,554 \\
\hline TGGCCCCAGGTGCCACC & Hs.268571 & Apolipoprotein C-I & 717 & 16,192 \\
\hline АСТТТTТСААААААААА & & Multiple match & 575 & 12,986 \\
\hline CTGGCCTCCCAATAAAG & Hs.73849 & Apolipoprotein C-III & 535 & 12,082 \\
\hline AAAACATTCTCCTCCGC & & $16 \mathrm{~S}$ ribosomal RNA, mitochondrial & 520 & 11,743 \\
\hline TGTGGAGAGCCTGGTTT & Hs. 237658 & Apolipoprotein A-II & 510 & 11,518 \\
\hline TTCATACACCTATCCCC & & NADH dehydrogenase subunit 4 , mitochondrial & 438 & 9,892 \\
\hline CGACCCCACGCCACCCC & Hs. 110675 & Apolipoprotein E & 280 & 6,323 \\
\hline CCCATCGTCCTAGAATT & & Cytochrome $\mathrm{c}$ oxidase subunit II, mitochondrial & 258 & 5,827 \\
\hline CACTACTCACCAGACGC & & Cytochrome $b$, mitochondrial & 252 & 5,691 \\
\hline CCTGTAATCCCAGCACT & & Multiple match & 222 & 5,014 \\
\hline AGGTGGCAAGAAATGGG & & $12 \mathrm{~S}$ ribosomal RNA, mitochondrial & 221 & 4,991 \\
\hline TGGACGCGCTGCGCACG & Hs.93194 & Apolipoprotein A-I & 218 & 4,923 \\
\hline CTAAGGTGGTTTTCAGA & Hs. 1252 & Apolipoprotein H (beta-2-glycoprotein I) & 213 & 4,810 \\
\hline GTGACCACGGGTGACGG & & ESTs & 201 & 4,539 \\
\hline TAACCAAGAGTATTCCA & Hs.427202 & Transthyretin (prealbumin, amyloidosis type I) & 192 & 4,336 \\
\hline GTGAAACCCCGTCTCTA & & Multiple match & 190 & 4,291 \\
\hline
\end{tabular}

We included the transcripts encoded by mitochondrial DNA in the library. The corresponding tags per million are shown to allow comparisons 


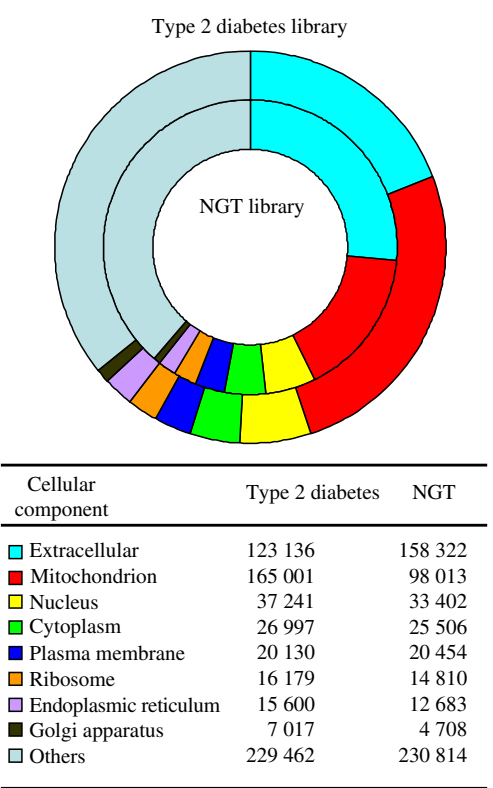

Fig. 1 Distribution of the cellular components in the type 2 diabetes and the NGT libraries. The cellular components of all identified transcripts were gathered by Gene Ontology (see Materials and methods). The tag count for the gene was added to the corresponding class (some transcripts bear several components in different classes). The cumulative tag counts for each class are represented here in tags per million

(Fig. 2a). Of these transcripts, $36(68 \%)$ were upregulated in the type 2 diabetes library. The comparison with total cumulated tag counts showed that the overall expression level of transcripts for OXPHOS was 1.7 times higher in the type 2 diabetes library than in the NGT library (7,933 vs 4,748 tags; $p<0.00001)$. Expression profiles of 53 OXPHOS genes in SAGE libraries are shown in ESM Table 1.

We detected two rRNAs and 11 mRNAs encoded by mitochondria in the two libraries (Fig. 2b). Both $12 \mathrm{~S}$ and $16 \mathrm{~S}$ rRNAs were upregulated in the type 2 diabetes library. Of 11 mRNAs, seven were upregulated in the type 2 diabetes library. A comparison using total cumulated tag counts showed that mitochondrial rRNAs were 3.5 -fold overexpressed and mRNAs were 1.2-fold over-expressed in the type 2 diabetes library $(64,905$ vs 18,485 tags and 74,505 vs 61,040 tags, respectively; $p<0.00001$ for both comparisons).

\section{Expression of genes involved in OXPHOS and glucose} metabolism by real-time quantitative PCR Using real-time PCR analysis, we reconfirmed the result of SAGE analysis for OXPHOS gene expression. The following representative OXPHOS genes were significantly upregulated in patients with type 2 diabetes: NADH dehydrogenase (ubiquinone) 1 alpha subcomplex, 6, $14 \mathrm{kDa}$ (NDUFA6) (Complex I), succinate dehydrogenase complex, subunit $\mathrm{C}$, integral membrane protein, $15 \mathrm{kDa}(S D H C)$ (Complex II), ubiquinol-cytochrome $\mathrm{c}$ reductase binding protein $(U Q C R B$, formerly known as $Q P C$ ) (Complex III), cytochrome c
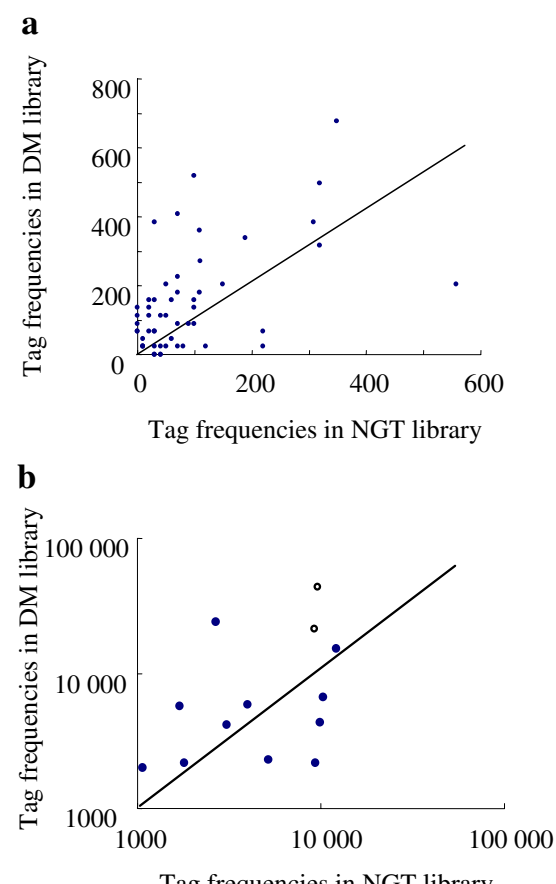

Fig. 2 Expression of transcripts involved in oxidative phosphorylation $(O X P H O S)$ in each serial analysis of gene expression ( $S A G E)$ library. SAGE tag frequencies of each transcript are shown in tags per million. a Fifty-three nuclear transcripts involved in OXPHOS were detected in the two libraries; 36 (68\%) were upregulated in the type 2 diabetes $(D M)$ library. Comparison with total cumulated tag counts showed that the overall expression level of transcripts for OXPHOS was 1.7 times higher in the type 2 diabetes library than in the NGT library (7933 vs 4748 tags $p<0.00001)$. b Thirteen mitochondrial transcripts were detected in the two libraries. The distribution of SAGE tags was plotted on a logarithmic scale. Two mitochondrial rRNAs and 11 mitochondrial mRNAs were expressed. $12 \mathrm{~S}$ and $16 \mathrm{~S}$ rRNAs were upregulated in the type 2 diabetes $(D M)$ library. Of 11 mRNAs, seven were upregulated in the type 2 diabetes library. Comparison with total cumulated tag counts showed that mitochondrial rRNAs were 3.5-fold over-expressed and mRNAs were 1.2-fold over-expressed in the type 2 diabetes library (64905 vs 18485 tags, 74505 vs 61040 tags $p<0.00001)$. Blue symbols, mRNA; open symbols, rRNA

oxidase subunit IV isoform 1 (COX4i1) (Complex IV), and ATP synthase, $\mathrm{H}^{+}$transporting, mitochondrial $\mathrm{F}$ 1 complex, beta polypeptide (ATP5B) (Complex V) (see ESM Fig. 1). Expression of representative genes for hepatic gluconeogenesis such as PPARGC1A, tribbles homolog 3 (Drosophila) (TRIB3, also known as TRB-3), mitochondrial phosphoenolpyruvate carboxykinase 2 (PCK2), and solute carrier family 2 (facilitated glucose transporter), member 2 ( $S L C 2 A 2$, also known as GLUT2) was significantly upregulated in the type 2 diabetes SAGE library $(p<0.001)$. Tag frequencies to other key molecules such as the genes encoding $H N F 4 A$, glucocorticoid receptors, glucose-6-phosphatase $(G 6 P C)$ and cAMP responsive element binding protein were not detected in the two libraries. Expression was also confirmed by real-time PCR (ESM Fig. 2). Expression of PPARGC1A was confirmed to be upregulated 
in the type 2 diabetes library (ESM Fig. 2a). The expression of genes encoding mitochondrial gluconeogenic key enzyme $P C K 2$ and cytosol enzyme $P C K 1$ was significantly upregulated in the type 2 diabetes library $(p<0.005$, ESM Fig. $2 \mathrm{~b}$ and c). Upregulation of those genes may provide a molecular basis for elevated gluconeogenesis in the livers of patients with type 2 diabetes.

Pathway analysis of genes involved in OXPHOS by DNA chip analysis We reconfirmed the results of SAGE and real-time PCR for genes involved in OXPHOS by using DNA chip analysis in our independent reference subject groups. Gene list comparison using the Biometric Research Branch (BRB) array tool (http://www.linus.nci. nih.gov/BRB-ArrayTools.html) revealed that the 114 genes involved in OXPHOS were coordinately upregulated in the livers of patients with type 2 diabetes $(p<$ 0.00005 , Fig. 3). Expression profiles of each OXPHOS gene in DNA chip analysis are shown in ESM Table 2. In the livers of patients with type 2 diabetes, OXPHOS genes encoding proteins for respiratory complexes I, II, III, IV and $\mathrm{V}$ were upregulated.

Univariate linear regression analysis to assess the contribution of clinical parameters to OXPHOS mean
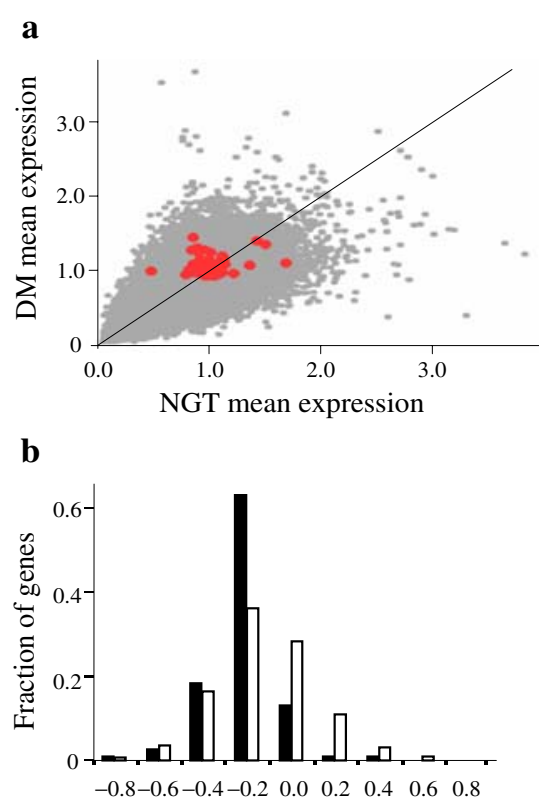

Mean expression difference (NGT-DM)

Fig. 3 Pathway analysis of genes involved in OXPHOS by DNA chip analysis. a The mean expression of all genes (grey dots) and of genes involved in OXPHOS (red dots) was plotted for individuals with type 2 diabetes $(D M)$ versus those with NGT. b Histogram of mean gene expression level differences between individuals with type 2 diabetes (DM) and NGT, using the data from all genes (white bars) and OXPHOS genes (black bars). Gene list comparison using BRB array tool revealed that 114 genes involved in OXPHOS were coordinately upregulated in livers of patients with type 2 diabetes $(p<0.00005)$ centroid To evaluate the correlation between expression of the genes involved in OXPHOS and clinical or biochemical parameters of individuals with type 2 diabetes and NGT, we computed the mean centroid of OXPHOS genes (Table 5). Mootha et al. reported that the mean centroid of OXPHOS genes in the skeletal muscle was correlated with total-body aerobic capacity [7]. In our analysis, the mean centroid of OXPHOS genes in the liver was significantly correlated with fasting plasma glucose level (Table 5 and Fig. 4a). In addition, the mean centroid of OXPHOS genes had a tendency to correlate negatively with markers of insulin resistance such as metabolic clearance rate and quantitative insulin sensitivity check index (Fig. 4b and c).

Multivariate linear analysis to assess the contribution of mean centroid of OXPHOS and clinical parameters to fasting plasma glucose level Because fasting plasma glucose levels were correlated with the mean centroid of OXPHOS genes, we hypothesised that the upregulation of genes involved in OXPHOS in the liver contributes to fasting hyperglycaemia in patients with type 2 diabetes. To test this hypothesis, we generated multivariate linear regression models using fasting glucose level as the dependent variable, and the mean centroid of OXPHOS together with clinical parameters as independent variables. As shown in Table 6, we found that the mean centroid of genes involved in OXPHOS was a predictor of fasting plasma glucose levels, independently of age, BMI, insulin resistance, and fasting insulin levels.

Table 5 Univariate linear regression analysis to assess the contribution of clinical parameters to OXPHOS mean centroid

\begin{tabular}{|c|c|c|}
\hline & \multicolumn{2}{|c|}{ OXPHOS mean centroid } \\
\hline & $p$ value & $r^{2}$ \\
\hline Age & 0.897 & 0.00 \\
\hline BMI & 0.144 & 0.13 \\
\hline Fasting plasma glucose & $0.015^{*}$ & 0.32 \\
\hline $\mathrm{HbA}_{1 \mathrm{c}}$ & $0.092 * *$ & 0.20 \\
\hline Fasting IRI & 0.208 & 0.12 \\
\hline HOMA-IR & 0.114 & 0.21 \\
\hline QUICKI & $0.075 * *$ & 0.22 \\
\hline Glucose metabolic clearance rate & $0.055^{* *}$ & 0.43 \\
\hline AST & 0.969 & 0.00 \\
\hline ALT & 0.986 & 0.00 \\
\hline Total cholesterol & 0.411 & 0.04 \\
\hline Triacylglycerol & 0.942 & 0.00 \\
\hline HDL & 0.619 & 0.02 \\
\hline
\end{tabular}

IRI Immunoreactive insulin, HOMA-IR homeostasis model assessment, QUICKI quantitative insulin sensitivity check index, $A S T$ aspartate transaminase, $A L T$ alanine aminotransferase $* p<0.05,{ }^{* *} p<0.1$ 

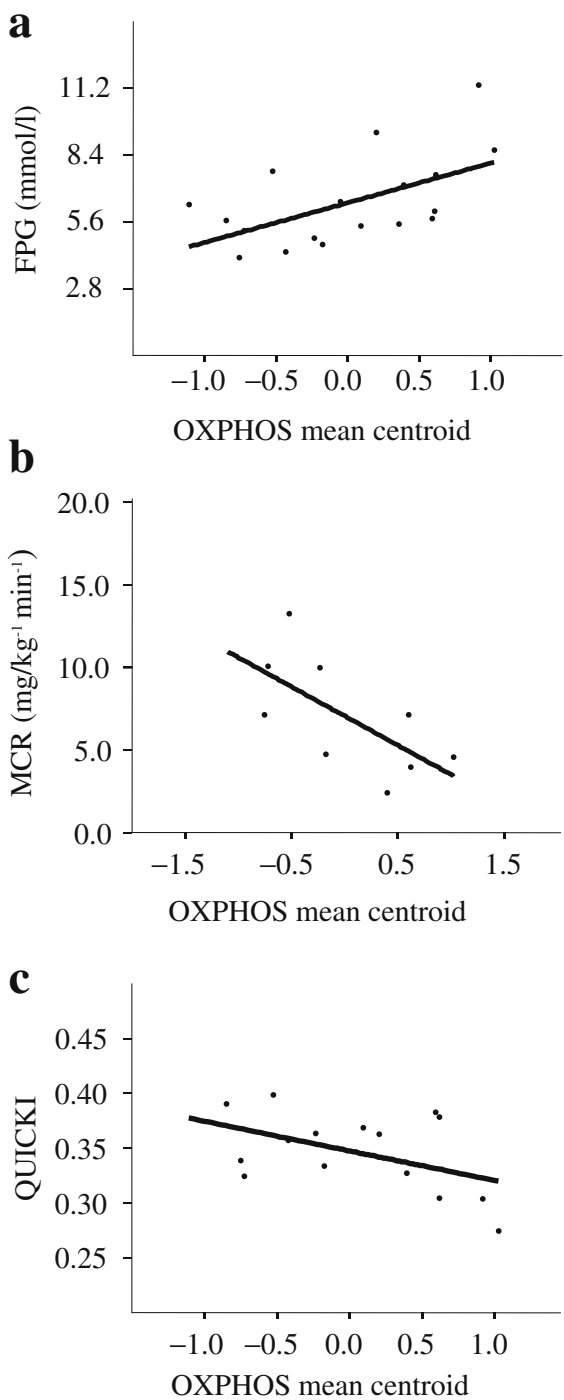

Fig. 4 Correlation between the mean centroid of $O X P H O S$ genes and fasting plasma glucose $(F P G)($ a), glucose metabolic clearance rate $(M C R)(\mathbf{b})$ and quantitative insulin sensitivitycheck index (QUICKI) (c). $r^{2}=0.32$ (a), 0.43 (b), 0.22 (c). $p=0.015$ (a), 0.055 (b), 0.075 (c)

Table 6 Multivariate linear analysis to assess the contribution of mean centroid of OXPHOS and clinical parameters to fasting plasma glucose level

\begin{tabular}{llll}
\hline & \multicolumn{2}{l}{ Fasting plasma glucose } \\
\cline { 2 - 4 } & Coefficient & $t$ statistic & $p$ value \\
\hline Age & 1.089 & 1.788 & 0.107 \\
BMI & -3.340 & -2.576 & $0.030^{*}$ \\
QUICKI & -780.282 & -1.899 & 0.090 \\
Fasting IRI & 1.8876 & -1.520 & 0.163 \\
Mean centroid of OXPHOS & 30.338 & 2.396 & $0.040^{*}$ \\
\hline
\end{tabular}

QUICKI Quantitative insulin sensitivity check index, IRI immunoreactive insulin

$* p<0.05$
Correlation between OXPHOS mean centroid and gene expression for transcriptional factors and cofactors We next investigated regulatory factors that control gene expression for OXPHOS. We analysed gene expression for transcriptional factors or nuclear co-activators that are reported to be associated with OXPHOS or energy homeostasis. The genes analysed included: PPARGC1A, PPARGC1B [17], nuclear respiratory factor 1 (NRFl) [17], peroxisome proliferator-activated receptors (PPARs), estrogen related receptor- $\alpha(E S R R A)$ [18], thyroid receptor $\alpha(T H R A)$ [19], steroid receptor co-activators-1 (NCOA1, also known as $S R C-1)$ [20] and transcriptional intermediary factor-2 (NCOA2, also known as TIF-2) [20]. As a result, gene expression for PPARG, ESRRA, NCOA1, THRA and NCOA2 was significantly correlated with the mean centroid of OXPHOS genes (Fig. 5). Surprisingly, gene expression of PPARGC1A and PPARGC1B (data not shown) did not correlate with the mean centroid of OXPHOS genes (Fig. 5f).

\section{Discussion}

The liver, an important glucose-releasing organ, plays a central role in glucose homeostasis and contributes to the development of type 2 diabetes. Several reports have demonstrated a reduction of genes involved in OXPHOS in the skeletal muscle of patients with type 2 diabetes [6,7], and raise the question as to whether this reduction is also seen in the liver of these patients. However, there are no reports of comprehensive gene expression of OXPHOS in the human liver, mainly because of the difficulty of obtaining liver samples from humans with diabetes. Previously, we reported that genes involved in systemic vascular complications are differentially expressed in the livers of patients with type 2 diabetes [21]. We report here the first comprehensive analysis of upregulated expression of genes involved in OXPHOS in the livers of humans with type 2 diabetes; no comparable findings have been shown in previous reports of animal or cellular experiments.

In our two analyses, there were no significant differences in BMI between subjects with type 2 diabetes and those with NGT. Unlike Western subjects, Japanese patients with type 2 diabetes are characterised by impaired insulin secretion rather than insulin resistance. Thus, obesity is not as severe in these patients; in fact, some Japanese patients with type 2 diabetes are lean. Therefore, the hepatic gene expresion profiles we obtained in the present study are more closely associated with type 2 diabetes itself than with obesity. In addition, none of the subjects in the two analyses experienced body weight loss or gain before liver biopsies, and no patients in SAGE analysis suffered from cancerinduced malnutrition or body weight loss. Thus, we believe that the influence of body weight change on hepatic gene 
Fig. 5 Correlation of gene expression between the mean centroid of OXPHOS and transcriptional factors or cofactors. PPARG, peroxisome proliferator-activated receptor- $\gamma$; ESRRA, estrogen related receptor- $\alpha$; NCOA1 (also known as $S R C-1)$, steroid receptor coactivator-1; THRA, thyroid hormone receptor- $\alpha$; NCOA2 (also known as $T I F-2)$, transcriptional intermediary factor-2; PPARGC1A, peroxisome proliferator-activated receptor- $\gamma$ coactivator $1 \alpha$. $r^{2}=0.38$ (a), 0.29 (b), 0.30 (c), $r^{2}=0.41(\mathbf{d}), 0.54(\mathbf{e}), 0.06(\mathbf{f})$. $p=0.006$ (a), 0.022 (b), 0.018 (c) 0.004 (d); $p<0.0001$ (e); $p>0.05$ (f) a

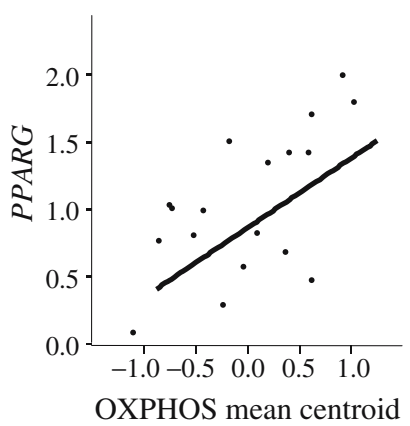

d

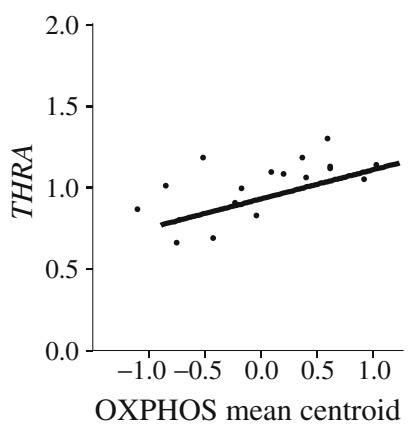

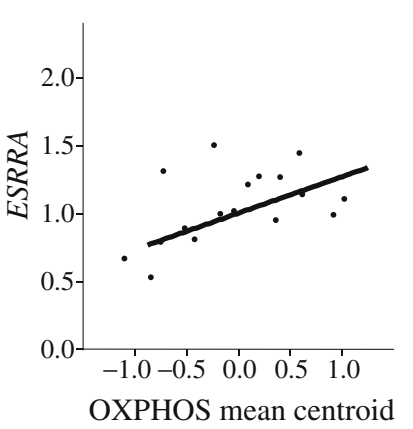

e

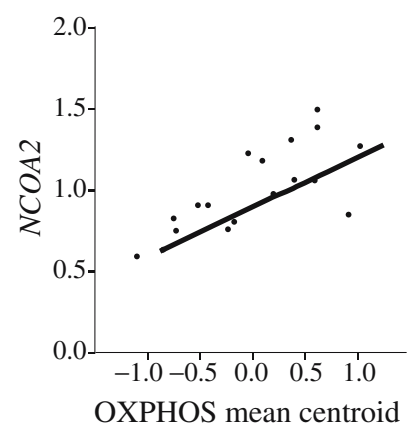

c

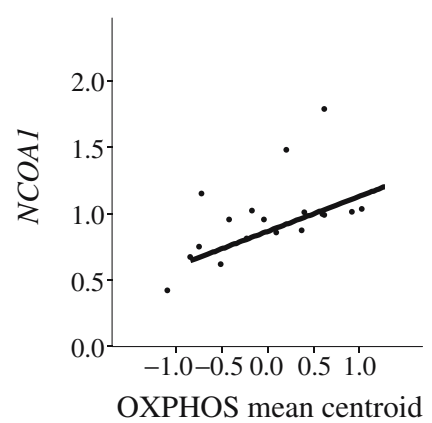

f

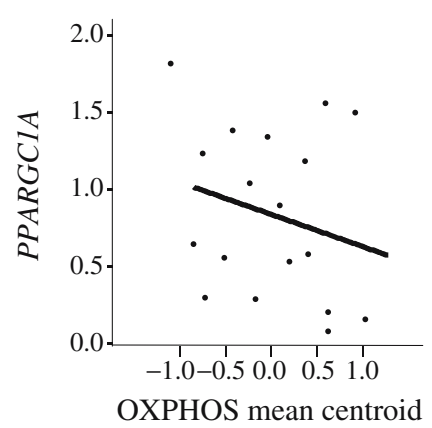

expression profiles for genes involved in OXPHOS can be ruled out in our study.

We constructed SAGE libraries from NGT and type 2 diabetic patients with different procedures: LongSAGE for the type 2 diabetes library and short SAGE for the NGT library. The LongSAGE method, which generates tags with a length of 21 bases, theoretically facilitates tag-to-gene assignments. In fact, more tags could be assigned to a single unique gene in our type 2 diabetes library by LongSAGE than in the NGT library by short SAGE (type 2 diabetes $73 \%$ vs NGT 65\%). However, stochastically, the amount of tags for individual genes detected by short SAGE and LongSAGE should be equal when analysing the same sample. In fact, we confirmed that the type 2 diabetes:NGT ratio of OXPHOS genes in SAGE was approximately equal to that in real-time PCR (data not shown). Therefore, we believe that the different SAGE methods did not influence the conclusion of our study.

The transcripts corresponding to the ontology class 'mitochondria' were 1.7-fold, and those of mitochondrial rRNAs 3.5-fold over-expressed in the type 2 diabetes SAGE library. In addition, nuclear and mitochondrial genes involved in OXPHOS were upregulated in the type 2 diabetes library. Subunits of respiratory complexes in the mitochondria were encoded by both nuclear and mitochondrial DNA [22]. However, the limited coding capacity of mitochondrial DNA means that nuclear genes make a major contribution to OXPHOS. For example, the interaction between nuclear factor PPARGC1A and NRF1 regulates mitochondrial gene transcription through the upregulation of mitochondrial transcription factor A [17]. Our results suggest that the upregulation of nuclear and mitochondrial genes involved in OXPHOS in type 2 diabetic livers is due to the overproduction of several nuclear factors, such as PPARGC1A, ESRRA and THRA.

One limitation of our study was that the analysis was performed only at the gene transcriptional levels. Because the liver samples from patients with type 2 diabetes were limited, we were unable to perform functional or structural analyses. Further studies on the alteration of mitochondrial bioenergetic capacity and morphology are necessary to evaluate hepatic mitochondrial function in patients with type 2 diabetes. However, our results suggest that the function of OXPHOS is upregulated in the type 2 diabetic liver, because mitochondrial rRNAs were 3.5-fold over-expressed. George et al. have reported that high ATP levels strongly stimulate mitochondrial rRNA synthesis in isolated human mitochondria [23]. These results suggest that, in the liver of patients with type 2 diabetes, ATP production is activated through the upregulation of genes involved in OXPHOS, which stimulates rRNA synthesis and activates energy metabolism. Our results correspond with rat studies by Ferreira et al. [24, 25], demonstrating that the efficiency of the OXPHOS system is elevated in the liver of Goto-Kakizaki rats, an animal model of type 2 
diabetes [24], whereas that in animal models of type 1 diabetes induced by streptozotocin is lowered [26-28].

We demonstrated that expression of genes involved in OXPHOS can predict fasting plasma glucose levels, independently of age, BMI and insulin resistance markers. Our findings suggest that upregulation of genes involved in OXPHOS in type 2 diabetic liver is associated with fasting hyperglycaemia. However, it is not clear whether the upregulation of OXPHOS genes in type 2 diabetic livers is a consequence or a cause of hyperglycaemia. In endothelial cells, which have an insulin-independent glucose uptake system like hepatocytes, treatment with glucose was reported to activate OXPHOS in the mitochondria with ease [29]. Similarly, therefore, influx of high glucose levels into hepatocytes could stimulate expression of genes involved in OXPHOS in the liver. Inversely, the upregulation of genes involved in OXPHOS may cause hyperglycaemia, because gluconeogenic enzymes such as PEPCK require ATP for their catalytic actions. The upregulation of genes involved in OXPHOS may contribute to hyperglycaemia through the supply of ATP for gluconeogenic enzymes. The glucose-lowering drug metformin is reported to restrain hepatic gluconeogenesis through inhibition of the respiratory chain $1[30,31]$. Our analysis is compatible with these reports on the pharmacological effect of metformin. The upregulation of genes involved in OXPHOS in the liver may therefore be a therapeutic target for the treatment of type 2 diabetes.

A reduction in expression of genes involved in OXPHOS has been reported in type 2 diabetic skeletal muscle $[6,7]$. This reduction is related to impaired oxidative capacity and accumulation of triacylglycerol in the skeletal muscle, which correlates with insulin resistance [5]. On the other hand, lipid accumulation in the liver is also observed in many patients with type 2 diabetes. Generally, in response to increased fatty acid synthesis, the mitochondrial $\beta$-oxidation pathway is also upregulated. Activation of $\beta$-oxidation of fatty acids distributes $\mathrm{FADH}_{2}, \mathrm{NADH}$ and acyl-CoA to mitochondrial OXPHOS, leading to OXPHOS activation. Thus, the liver might compensate for steatosis by increasing fatty acid $\beta$-oxidation and activating OXPHOS in patients with type 2 diabetes. However, such increased oxidative capacity in the type 2 diabetic liver may not be enough to stop hepatic steatosis. Further study to elucidate the significance of OXPHOS in the development of fatty liver is needed.

PPARGC1A is a nuclear hormone receptor coactivator, which powerfully induces and coordinates expression of genes involved in hepatic gluconeogenesis [32]. In the current study, elevated expression of PPARGC1A was demonstrated in the liver of patients with type 2 diabetes. However, expression of PPARGC1A did not correlate with that of genes involved in OXPHOS. Mootha et al. identified a subset of OXPHOS genes coordinately regulated by
PPARGC1A in human skeletal muscle and termed this subset OXPHOS-CR (oxidative phosphorylation coregulated). However, our clustering analysis showed that expression of Mootha's OXPHOS-CR genes had no coregulation in human liver samples (data not shown). Thus, PPARGC1A may not be the only master gene to regulate expression of genes involved in OXPHOS in the liver. The regulatory mechanism for genes involved in OXPHOS in the liver may be much different from that in the skeletal muscle. Actually, the regulation of genes for OXPHOS in the liver of patients with type 2 diabetes appears to be a mirror image of that of the skeletal muscle in patients with type 2 diabetes, in which genes involved in OXPHOS are downregulated. The differential gene expression for OXPHOS between the liver and skeletal muscle in type 2 diabetes may be derived from the difference in the glucose uptake systems of hepatocytes and myocytes. Because hepatocytes have an insulin-independent glucose uptake system, excessive glucose influx may activate intracellular OXPHOS easily in a diabetic condition. On the other hand, in myocytes with their insulin-dependent glucose uptake system, hyperglycaemia may not activate OXPHOS, even in a diabetic condition. Searching for the upstream master gene that regulates tissue-specific expression of genes involved in OXPHOS may become an important issue in elucidation of the mechanism of glucose homeostasis. Further comprehensive gene analysis may help identify candidate master genes that regulate OXPHOS genes in human liver and contribute to the pathology of type 2 diabetes.

In conclusion, genes involved in OXPHOS were coordinately upregulated with fasting hyperglycaemia in the livers of patients with type 2 diabetes. Elucidating the significance of hepatic OXPHOS will help understand the pathophysiology of type 2 diabetes, and help to discover novel therapeutic targets for its treatment.

Acknowledgements We thank G. Nishimura and K. Miwa from the Department of Surgery II, Kanazawa University, for generously providing the liver tissue samples.

Duality of interest None of the authors are aware of any duality of interest.

\section{References}

1. Michael MD, Kulkarni RN, Postic C et al (2000) Loss of insulin signaling in hepatocytes leads to severe insulin resistance and progressive hepatic dysfunction. Mol Cell 6:87-97

2. Lowell BB, Shulman GI (2005) Mitochondrial dysfunction and type 2 diabetes. Science 307:384-387

3. Kelley DE, He J, Menshikova EV et al (2002) Dysfunction of mitochondria in human skeletal muscle in type 2 diabetes. Diabetes 51:2944-2950

4. Petersen KF, Befroy D, Dufour S et al (2003) Mitochondrial dysfunction in the elderly: possible role in insulin resistance. Science 300:1140-1142 
5. Petersen KF, Dufour S, Befroy D et al (2004) Impaired mitochondrial activity in the insulin-resistant offspring of patients with type 2 diabetes. N Engl J Med 350:664-671

6. Patti ME, Butte AJ, Crunkhorn S et al (2003) Coordinated reduction of genes of oxidative metabolism in humans with insulin resistance and diabetes: potential role of PGC1 and NRF1. Proc Natl Acad Sci USA 100:8466-8471

7. Mootha VK, Lindgren CM, Eriksson KF et al (2003) PGC1alpha-responsive genes involved in oxidative phosphorylation are coordinately downregulated in human diabetes. Nat Genet 34:267-273

8. Yoon JC, Puigserver P, Chen G et al (2001) Control of hepatic gluconeogenesis through the transcriptional coactivator PGC-1. Nature 413:131-138

9. Saltiel AR, Kahn CR (2001) Insulin signalling and the regulation of glucose and lipid metabolism. Nature 414:799-806

10. Herzig S, Long F, Jhala US et al (2001) CREB regulates hepatic gluconeogenesis through the coactivator PGC-1. Nature 413:179183

11. Koo SH, Satoh H, Herzig S et al (2004) PGC-1 promotes insulin resistance in liver through PPAR-alpha-dependent induction of TRB-3. Nat Med 10:530-534

12. Velculescu VE, Zhang L, Vogelstein B et al (1995) Serial analysis of gene expression. Science 270:484-487

13. No authors listed (2003) Report of the expert committee on the diagnosis and classification of diabetes mellitus. Diabetes Care 26 (Suppl 1):S5-S20

14. Yamashita T, Hashimoto S, Kaneko S et al (2000) Comprehensive gene expression profile of a normal human liver. Biochem Biophys Res Commun 269:110-116

15. Yamashita T, Kaneko S, Hashimoto S et al (2001) Serial analysis of gene expression in chronic hepatitis $\mathrm{C}$ and hepatocellular carcinoma. Biochem Biophys Res Commun 282:647-654

16. Saha S, Sparks AB, Rago C et al (2002) Using the transcriptome to annotate the genome. Nat Biotechnol 20:508-512

17. Scarpulla RC (2002) Nuclear activators and coactivators in mammalian mitochondrial biogenesis. Biochim Biophys Acta 1576:1-14

18. Mootha VK, Handschin C, Arlow D et al (2004) Erralpha and Gabpa/b specify PGC-1alpha-dependent oxidative phosphorylation gene expression that is altered in diabetic muscle. Proc Natl Acad Sci USA 101:6570-6575

19. Wrutniak-Cabello C, Casas F, Cabello G (2001) Thyroid hormone action in mitochondria. J Mol Endocrinol 26:67-77
20. Picard F, Gehin M, Annicotte J et al (2002) SRC-1 and TIF2 control energy balance between white and brown adipose tissues. Cell 111:931-941

21. Takamura T, Sakurai M, Ota T et al (2004) Genes for systemic vascular complications are differentially expressed in the livers of type 2 diabetic patients. Diabetologia 47:638-647

22. Garesse R, Vallejo CG (2001) Animal mitochondrial biogenesis and function: a regulatory cross-talk between two genomes. Gene 263:1-16

23. Gaines G, Rossi C, Attardi G (1987) Markedly different ATP requirements for rRNA synthesis and mtDNA light strand transcription versus mRNA synthesis in isolated human mitochondria. J Biol Chem 262:1907-1915

24. Ferreira FM, Palmeira CM, Seica R et al (1999) Alterations of liver mitochondrial bioenergetics in diabetic Goto-Kakizaki rats. Metabolism 48:1115-1119

25. Ferreira FM, Seica R, Oliveira PJ et al (2003) Diabetes induces metabolic adaptations in rat liver mitochondria: role of coenzyme Q and cardiolipin contents. Biochim Biophys Acta 1639:113-120

26. Memon RA, Mohan C, Bessman SP (1995) Impaired mitochondrial protein synthesis in streptozotocin diabetic rat hepatocytes. Biochem Mol Biol Int 37:627-634

27. Kaplan RS, Oliveira DL, Wilson GL (1990) Streptozotocininduced alterations in the levels of functional mitochondrial anion transport proteins. Arch Biochem Biophys 280:181-191

28. Ulicna O, Volkovova K, Istvanova B (1996) Bioenergetics of liver mitochondria in rats in experimental insulin-dependent diabetes. [Article in Slovak] Bratisl Lek Listy 97:619-624

29. Nishikawa T, Edelstein D, Du XL et al (2000) Normalizing mitochondrial superoxide production blocks three pathways of hyperglycaemic damage. Nature 404:787-790

30. Guigas B, Detaille D, Chauvin C et al (2004) Metformin inhibits mitochondrial permeability transition and cell death: a pharmacological in vitro study. Biochem J 382:877-884

31. Owen MR, Doran E, Halestrap AP (2000) Evidence that metformin exerts its anti-diabetic effects through inhibition of complex 1 of the mitochondrial respiratory chain. Biochem $\mathrm{J}$ 348:607-614

32. Puigserver P, Spiegelman BM (2003) Peroxisome proliferatoractivated receptor-gamma coactivator 1 alpha (PGC-1 alpha): transcriptional coactivator and metabolic regulator. Endocr Rev 24:78-90 